\title{
Vision Transformers for Dense Prediction
}

René Ranftl

\author{
Alexey Bochkovskiy \\ Intel Labs \\ rene.ranftl@intel.com
}

Vladlen Koltun

\begin{abstract}
We introduce dense vision transformers, an architecture that leverages vision transformers in place of convolutional networks as a backbone for dense prediction tasks. We assemble tokens from various stages of the vision transformer into image-like representations at various resolutions and progressively combine them into full-resolution predictions using a convolutional decoder. The transformer backbone processes representations at a constant and relatively high resolution and has a global receptive field at every stage. These properties allow the dense vision transformer to provide finer-grained and more globally coherent predictions when compared to fully-convolutional networks. Our experiments show that this architecture yields substantial improvements on dense prediction tasks, especially when a large amount of training data is available. For monocular depth estimation, we observe an improvement of up to $28 \%$ in relative performance when compared to a state-of-theart fully-convolutional network. When applied to semantic segmentation, dense vision transformers set a new state of the art on ADE20K with $49.02 \%$ mIoU. We further show that the architecture can be fine-tuned on smaller datasets such as NYUv2, KITTI, and Pascal Context where it also sets the new state of the art. Our models are available at https://github.com/intel-isl/DPT
\end{abstract}

\section{Introduction}

Virtually all existing architectures for dense prediction are based on convolutional networks [6, 31, 34, 42, 49, 50, 53]. The design of dense prediction architectures commonly follows a pattern that logically separates the network into an encoder and a decoder. The encoder is frequently based on an image classification network, also called the backbone, that is pretrained on a large corpus such as ImageNet [9]. The decoder aggregates features from the encoder and converts them to the final dense predictions. Architectural research on dense prediction frequently focuses on the decoder and its aggregation strategy [6, 7, 50, 53]. However, it is widely recognized that the choice of backbone architecture has a large influence on the capabilities of the overall model, as any information that is lost in the encoder is impossible to recover in the decoder.

Convolutional backbones progressively downsample the input image to extract features at multiple scales. Downsampling enables a progressive increase of the receptive field, the grouping of low-level features into abstract highlevel features, and simultaneously ensures that memory and computational requirements of the network remain tractable. However, downsampling has distinct drawbacks that are particularly salient in dense prediction tasks: feature resolution and granularity are lost in the deeper stages of the model and can thus be hard to recover in the decoder. While feature resolution and granularity may not matter for some tasks, such as image classification, they are critical for dense prediction, where the architecture should ideally be able to resolve features at or close to the resolution of the input image.

Various techniques to mitigate the loss of feature granularity have been proposed. These include training at higher input resolution (if the computational budget permits), dilated convolutions [49] to rapidly increase the receptive field without downsampling, appropriately-placed skip connections from multiple stages of the encoder to the decoder [31], or, more recently, by connecting multiresolution representations in parallel throughout the network [42]. While these techniques can significantly improve prediction quality, the networks are still bottlenecked by their fundamental building block: the convolution. Convolutions together with non-linearities form the fundamental computational unit of image analysis networks. Convolutions, by definition, are linear operators that have a limited receptive field. The limited receptive field and the limited expressivity of an individual convolution necessitate sequential stacking into very deep architectures to acquire sufficiently broad context and sufficiently high representational power. This, however, requires the production of many intermediate representations that require a large amount of 
memory. Downsampling the intermediate representations is necessary to keep memory consumption at levels that are feasible with existing computer architectures.

In this work, we introduce the dense prediction transformer (DPT). DPT is a dense prediction architecture that is based on an encoder-decoder design that leverages a transformer as the basic computational building block of the encoder. Specifically, we use the recently proposed vision transformer (ViT) [11] as a backbone architecture. We reassemble the bag-of-words representation that is provided by ViT into image-like feature representations at various resolutions and progressively combine the feature representations into the final dense prediction using a convolutional decoder. Unlike fully-convolutional networks, the vision transformer backbone foregoes explicit downsampling operations after an initial image embedding has been computed and maintains a representation with constant dimensionality throughout all processing stages. It furthermore has a global receptive field at every stage. We show that these properties are especially advantageous for dense prediction tasks as they naturally lead to fine-grained and globally coherent predictions.

We conduct experiments on monocular depth estimation and semantic segmentation. For the task of general-purpose monocular depth estimation [30], where large-scale training data is available, DPT provides a performance increase of more than $28 \%$ when compared to the top-performing fully-convolutional network for this task. The architecture can also be fine-tuned to small monocular depth prediction datasets, such as NYUv2 [35] and KITTI [15], where it also sets the new state of the art. We provide further evidence of the strong performance of DPT using experiments on semantics segmentation. For this task, DPT sets a new state of the art on the challenging ADE20K [54] and Pascal Context [26] datasets. Our qualitative results indicate that the improvements can be attributed to finer-grained and more globally coherent predictions in comparison to convolutional networks.

\section{Related Work}

Fully-convolutional networks [33, 34] are the prototypical architecture for dense prediction. Many variants of this basic pattern have been proposed over the years, however, all existing architectures adopt convolution and subsampling as their fundamental elements in order to learn multi-scale representations that can leverage an appropriately large context. Several works propose to progressively upsample representations that have been pooled at different stages [1, 23, 27, 31], while others use dilated convolutions [6, 7, 49] or parallel multi-scale feature aggregation at multiple scales [53] to recover fine-grained predictions while at the same time ensuring a sufficiently large context. More recent architectures maintain a high-resolution repre- sentation together with multiple lower-resolution representations throughout the network [37, 42].

Attention-based models [2] and in particular transformers [39] have been the architecture of choice for learning strong models for natural language processing (NLP) [4, 10, 24] in recent years. Transformers are set-to-set models that are based on the self-attention mechanism. Transformer models have been particularly successful when instantiated as high-capacity architectures and trained on very large datasets. There have been several works that adapt attention mechanisms to image analysis [3, 28, 29, 41, 52]. In particular, it has recently been demonstrated that a direct application of token-based transformer architectures that have been successful in NLP can yield competitive performance on image classification [11]. A key insight of this work was that, like transformer models in NLP, vision transformers need to be paired with a sufficient amount of training data to realize their potential.

\section{Architecture}

This section introduces the dense vision transformer. We maintain the overall encoder-decoder structure that has been successful for dense prediction in the past. We leverage vision transformers [11] as the backbone, show how the representation that is produced by this encoder can be effectively transformed into dense predictions, and provide intuition for the success of this strategy. An overview of the complete architecture is shown in Figure 1 (left).

Transformer encoder. On a high level, the vision transformer (ViT) [11] operates on a bag-of-words representation of the image [36]. Image patches that are individually embedded into a feature space, or alternatively deep features extracted from the image, take the role of "words". We will refer to embedded "words" as tokens throughout the rest of this work. Transformers transform the set of tokens using sequential blocks of multi-headed self-attention (MHSA) [39], which relate tokens to each other to transform the representation.

Importantly for our application, a transformer maintains the number of tokens throughout all computations. Since tokens have a one-to-one correspondence with image patches, this means that the ViT encoder maintains the spatial resolution of the initial embedding throughout all transformer stages. Additionally, MHSA is an inherently global operation, as every token can attend to and thus influence every other token. Consequently, the transformer has a global receptive field at every stage after the initial embedding. This is in stark contrast to convolutional networks, which progressively increase their receptive field as features pass through consecutive convolution and downsampling layers.

More specifically, ViT extracts a patch embedding from the image by processing all non-overlapping square patches 


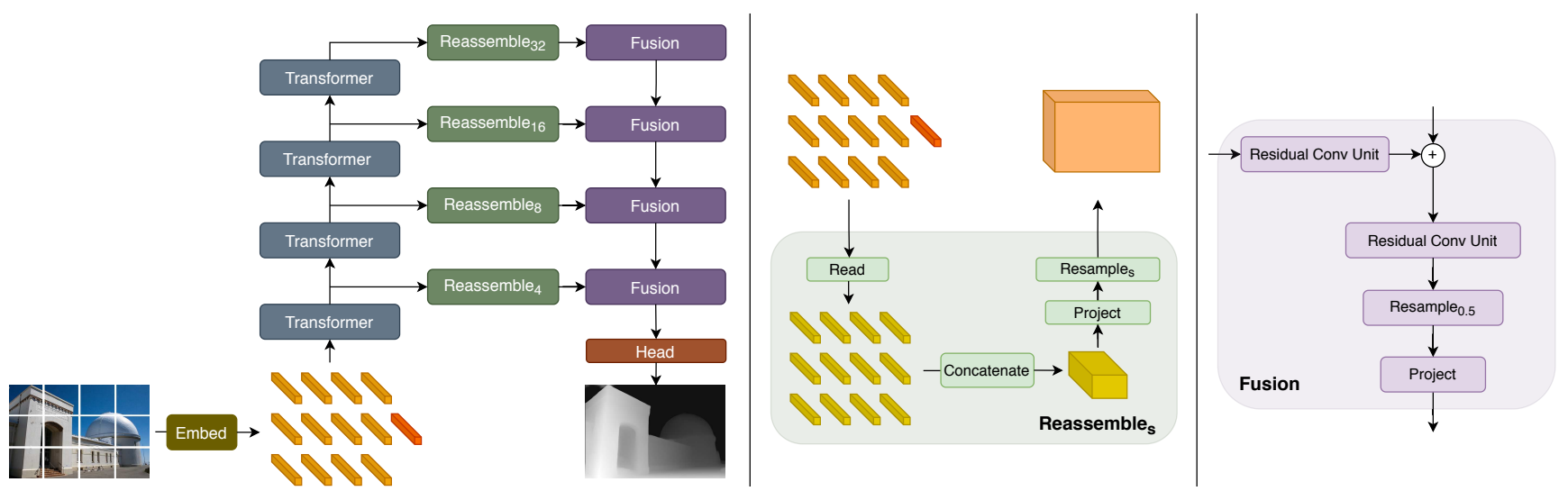

Figure 1. Left: Architecture overview. The input image is transformed into tokens (orange) either by extracting non-overlapping patches followed by a linear projection of their flattened representation (DPT-Base and DPT-Large) or by applying a ResNet-50 feature extractor (DPT-Hybrid). The image embedding is augmented with a positional embedding and a patch-independent readout token (red) is added. The tokens are passed through multiple transformer stages. We reassemble tokens from different stages into an image-like representation at multiple resolutions (green). Fusion modules (purple) progressively fuse and upsample the representations to generate a fine-grained prediction. Center: Overview of the Reassemble ${ }_{s}$ operation. Tokens are assembled into feature maps with $\frac{1}{s}$ the spatial resolution of the input image. Right: Fusion blocks combine features using residual convolutional units [23] and upsample the feature maps.

of size $p^{2}$ pixels from the image. The patches are flattened into vectors and individually embedded using a linear projection. An alternative, more sample-efficient, variant of ViT extracts the embedding by applying a ResNet50 [16] to the image and uses the pixel features of the resulting feature maps as tokens. Since transformers are set-to-set functions, they do not intrinsically retain the information of the spatial positions of individual tokens. The image embeddings are thus concatenated with a learnable position embedding to add this information to the representation. Following work in NLP, the ViT additionally adds a special token that is not grounded in the input image and serves as the final, global image representation which is used for classification. We refer to this special token as the readout token. The result of applying the embedding procedure to an image of size $H \times W$ pixels is a a set of $t^{0}=\left\{t_{0}^{0}, \ldots, t_{N_{p}}^{0}\right\}, t_{n}^{0} \in \mathbb{R}^{D}$ tokens, where $N_{p}=\frac{H W}{p^{2}}, t_{0}$ refers to the readout token, and $D$ is the feature dimension of each token.

The input tokens are transformed using $L$ transformer layers into new representations $t^{l}$, where $l$ refers to the output of the $l$-th transformer layer. Dosovitskiy et al. [11] define several variants of this basic blueprint. We use three variants in our work: ViT-Base, which uses the patch-based embedding procedure and features 12 transformer layers; ViT-Large, which uses the same embedding procedure and has 24 transformer layers and a wider feature size $D$; and ViT-Hybrid, which employs a ResNet50 to compute the image embedding followed by 12 transformer layers. We use patch size $p=16$ for all experiments. We refer the interested reader to the original work [11] for additional details on these architectures.

The embedding procedure for ViT-Base and ViT-Large projects the flattened patches to dimension $D=768$ and $D=1024$, respectively. Since both feature dimensions are larger than the number of pixels in an input patch, this means that the embedding procedure can learn to retain information if it is beneficial for the task. Features from the input patches can in principle be resolved with pixel-level accuracy. Similarly, the ViT-Hybrid architecture extracts features at $\frac{1}{16}$ the input resolution, which is twice as high as the lowest-resolution features that are commonly used with convolutional backbones.

Convolutional decoder. Our decoder assembles the set of tokens into image-like feature representations at various resolutions. The feature representations are progressively fused into the final dense prediction. We propose a simple three-stage Reassemble operation to recover image-like representations from the output tokens of arbitrary layers of the transformer encoder:

$$
\text { Reassemble }_{s}^{\hat{D}}(t)=\left(\text { Resample }_{s} \circ \text { Concatenate } \circ \operatorname{Read}\right)(t),
$$

where $s$ denotes the output size ratio of the recovered representation with respect to the input image, and $\hat{D}$ denotes the output feature dimension.

We first map the $N_{p}+1$ tokens to a set of $N_{p}$ tokens that is amenable to spatial concatenation into an image-like representation:

$$
\text { Read }: \mathbb{R}^{N_{p}+1 \times D} \rightarrow \mathbb{R}^{N_{p} \times D} .
$$

This operation is essentially responsible for appropriately handling the readout token. Since the readout token doesn't serve a clear purpose for the task of dense prediction, but could potentially still be useful to capture and distribute 
global information, we evaluate three different variants of this mapping:

$$
\operatorname{Read}_{\text {ignore }}(t)=\left\{t_{1}, \ldots, t_{N_{p}}\right\}
$$

simply ignores the readout token,

$$
\operatorname{Read}_{a d d}(t)=\left\{t_{1}+t_{0}, \ldots, t_{N_{p}}+t_{0}\right\}
$$

passes the information from the readout token to all other tokens by adding the representations, and

$$
\begin{aligned}
\operatorname{Read}_{\text {proj }}(t)=\left\{\operatorname{mlp}\left(\operatorname{cat}\left(t_{1}, t_{0}\right)\right)\right. & , \ldots, \\
& \left.\operatorname{mlp}\left(\operatorname{cat}\left(t_{N_{p}}, t_{0}\right)\right)\right\}
\end{aligned}
$$

passes information to the other tokens by concatenating the readout to all other tokens before projecting the representation to the original feature dimension $D$ using a linear layer followed by a GELU non-linearity [17].

After a Read block, the resulting $N_{p}$ tokens can be reshaped into an image-like representation by placing each token according to the position of the initial patch in the image. Formally, we apply a spatial concatenation operation that results in a feature map of size $\frac{H}{p} \times \frac{W}{p}$ with $D$ channels:

$$
\text { Concatenate }: \mathbb{R}^{N_{p} \times D} \rightarrow \mathbb{R}^{\frac{H}{p} \times \frac{W}{p} \times D} .
$$

We finally pass this representation to a spatial resampling layer that scales the representation to size $\frac{H}{s} \times \frac{W}{s}$ with $\hat{D}$ features per pixel:

$$
\text { Resample }_{s}: \mathbb{R}^{\frac{H}{p} \times \frac{W}{p} \times D} \rightarrow \mathbb{R}^{\frac{H}{s} \times \frac{W}{s} \times \hat{D}} .
$$

We implement this operation by first using $1 \times 1$ convolutions to project the input representation to $\hat{D}$, followed by a (strided) $3 \times 3$ convolution when $s \geq p$, or a strided $3 \times 3$ transpose convolution when $s<p$, to implement spatial downsampling and upsampling operations, respectively.

Irrespective of the exact transformer backbone, we reassemble features at four different stages and four different resolutions. We assemble features from deeper layers of the transformer at lower resolution, whereas features from early layers are assembled at higher resolution. When using ViT-Large, we reassemble tokens from layers $l=\{5,12,18,24\}$, whereas with ViT-Base we use layers $l=\{3,6,9,12\}$. We use features from the first and second ResNet block from the embedding network and stages $l=\{9,12\}$ when using ViT-Hybrid. Our default architecture uses projection as the readout operation and produces feature maps with $\hat{D}=256$ dimensions. We will refer to these architectures as DPT-Base, DPT-Large, and DPTHybrid, respectively.

We finally combine the extracted feature maps from consecutive stages using a RefineNet-based feature fusion block [23, 45] (see Figure1 (right)) and progressively upsample the representation by a factor of two in each fusion stage. The final representation size has half the resolution of the input image. We attach a task-specific output head to produce the final prediction. A schematic overview of the complete architecture is shown in Figure 1

Handling varying image sizes. Akin to fully-convolutional networks, DPT can handle varying image sizes. As long as the image size is divisible by $p$, the embedding procedure can be applied and will produce a varying number of image tokens $N_{p}$. As a set-to-set architecture, the transformer encoder can trivially handle a varying number of tokens. However, the position embedding has a dependency on the image size as it encodes the locations of the patches in the input image. We follow the approach proposed in [11] and linearly interpolate the position embeddings to the appropriate size. Note that this can be done on the fly for every image. After the embedding procedure and the transformer stages, both the reassemble and fusion modules can trivially handle a varying number of tokens, provided that the input image is aligned to the stride of the convolutional decoder (32 pixels).

\section{Experiments}

We apply DPT to two dense prediction tasks: monocular depth estimation and semantic segmentation. For both tasks, we show that DPT can significantly improve accuracy when compared to convolutional networks with a similar capacity, especially if a large training dataset is available. We first present our main results using the default configuration and show comprehensive ablations of different DPT configurations at the end of this section.

\subsection{Monocular Depth Estimation}

Monocular depth estimation is typically cast as a dense regression problem. It has been shown that massive metadatasets can be constructed from existing sources of data, provided that some care is taken in how different representations of depth are unified into a common representation and that common ambiguities (such as scale ambiguity) are appropriately handled in the training loss [30]. Since transformers are known to realize their full potential only when an abundance of training data is available, monocular depth estimation is an ideal task to test the capabilities of DPT.

Experimental protocol. We closely follow the protocol of Ranftl et al. [30]. We learn a monocular depth prediction network using a scale- and shift-invariant trimmed loss that operates on an inverse depth representation, together with the gradient-matching loss proposed in [22]. We construct a meta-dataset that includes the original datasets that were used in [30] (referred to as MIX 5 in that work) and extend it with with five additional datasets $([18,43,44,46,47])$. 


\begin{tabular}{|c|c|c|c|c|c|c|c|c|c|c|c|c|}
\hline \multicolumn{2}{|r|}{ Training set } & $\begin{array}{c}\text { DIW } \\
\text { WHDR }\end{array}$ & \multicolumn{2}{|c|}{$\begin{array}{l}\text { ETH3D } \\
\text { AbsRel }\end{array}$} & \multicolumn{2}{|c|}{$\begin{array}{c}\text { Sintel } \\
\text { AbsRel }\end{array}$} & \multicolumn{2}{|c|}{$\begin{array}{l}\text { KITTI } \\
\delta>1.25\end{array}$} & \multicolumn{2}{|c|}{$\begin{array}{c}\text { NYU } \\
\delta>1.25 \\
\end{array}$} & \multicolumn{2}{|c|}{$\begin{array}{c}\text { TUM } \\
\delta>1.25\end{array}$} \\
\hline$\overline{\text { DPT - Large }}$ & MIX 6 & $\mathbf{1 0 . 8 2}(-13.2 \%)$ & 0.089 & $(-31.2 \%)$ & 0.270 & $(-17.5 \%)$ & 8.46 & $(-64.6 \%)$ & 8.32 & $(-12.9 \%)$ & 9.97 & $(-30.3 \%)$ \\
\hline DPT - Hybrid & MIX 6 & $11.06 \quad(-11.2 \%)$ & 0.093 & $(-27.6 \%)$ & 0.274 & $(-16.2 \%)$ & 11.56 & $(-51.6 \%)$ & 8.69 & $(-9.0 \%)$ & 10.89 & $(-23.2 \%)$ \\
\hline MiDaS & MIX 6 & $12.95(+3.9 \%)$ & 0.116 & $(-10.5 \%)$ & 0.329 & $(+0.5 \%)$ & 16.08 & $(-32.7 \%)$ & 8.71 & $(-8.8 \%)$ & 12.51 & $(-12.5 \%)$ \\
\hline MiDaS [30] & MIX 5 & 12.46 & 0.129 & & 0.327 & & 23.90 & & 9.55 & & 14.29 & \\
\hline Li [22] & MD [22] & 23.15 & 0.181 & & 0.385 & & 36.29 & & 27.52 & & 29.54 & \\
\hline Li [21] & MC [21] & 26.52 & 0.183 & & 0.405 & & 47.94 & & 18.57 & & 17.71 & \\
\hline Wang 40 & WS 40] & 19.09 & 0.205 & & 0.390 & & 31.92 & & 29.57 & & 20.18 & \\
\hline Xian 45 & RW 45 & 14.59 & 0.186 & & 0.422 & & 34.08 & & 27.00 & & 25.02 & \\
\hline Casser 5 & $\mathrm{CS} 8$ & 32.80 & 0.235 & & 0.422 & & 21.15 & & 39.58 & & 37.18 & \\
\hline
\end{tabular}

Table 1. Comparison to the state of the art on monocular depth estimation. We evaluate zero-shot cross-dataset transfer according to the protocol defined in [30]. Relative performance is computed with respect to the original MiDaS model [30]. Lower is better for all metrics.

We refer to this meta-dataset as MIX 6. It contains about 1.4 million images and is, to the best of our knowledge, the largest training set for monocular depth estimation that has ever been compiled.

We use multi-objective optimization [32] together with Adam [19] and set a learning rate of $1 \mathrm{e}-5$ for the backbone and $1 \mathrm{e}-4$ for the decoder weights. The encoder is initialized with ImageNet-pretrained weights, whereas the decoder is initialized randomly. We use an output head that consists of 3 convolutional layers. The output head progressively halves the feature dimension and upsamples the predictions to the input resolution after the first convolutional layer (details in supplementary material). We disable batch normalization in the decoder, as we found it to negatively influence results for regression tasks. We resize the image such that the longer side is 384 pixels and train on random square crops of size 384 . We train for 60 epochs, where one epoch consists of 72,000 steps with a batch size of 16 . As the batch size is not divisible by the number of datasets, we construct a mini-batch by first drawing datasets uniformly at random before sampling from the respective datasets. We perform random horizontal flips for data augmentation. Similar to [30], we first pretrain on a well-curated subset of the data [45, 46, 47] for 60 epochs before training on the full dataset.

\begin{tabular}{lcccccc}
\hline & $\delta>1.25$ & $\delta>1.25^{2}$ & $\delta>1.25^{3}$ & AbsRel & RMSE & $\log 10$ \\
\hline DORN [13] & 0.828 & 0.965 & 0.992 & 0.115 & 0.509 & 0.051 \\
VNL [48] & 0.875 & 0.976 & 0.994 & 0.111 & 0.416 & 0.048 \\
BTS [20] & 0.885 & 0.978 & 0.994 & $\mathbf{0 . 1 1 0}$ & 0.392 & 0.047 \\
\hline DPT-Hybrid & $\mathbf{0 . 9 0 4}$ & $\mathbf{0 . 9 8 8}$ & $\mathbf{0 . 9 9 8}$ & $\mathbf{0 . 1 1 0}$ & $\mathbf{0 . 3 5 7}$ & $\mathbf{0 . 0 4 5}$ \\
\hline
\end{tabular}

Table 2. Evaluation on NYUv2 depth.

\begin{tabular}{lcccccc}
\hline & $\delta>1.25$ & $\delta>1.25^{2}$ & $\delta>1.25^{3}$ & AbsRel & RMSE & RMSE log \\
\hline DORN [13] & 0.932 & 0.984 & 0.994 & 0.072 & 2.626 & 0.120 \\
VNL [48] & 0.938 & 0.990 & 0.998 & 0.072 & 3.258 & 0.117 \\
BTS [20] & 0.956 & 0.993 & 0.998 & $\mathbf{0 . 0 5 9}$ & 2.756 & 0.096 \\
\hline DPT-Hybrid & $\mathbf{0 . 9 5 9}$ & $\mathbf{0 . 9 9 5}$ & $\mathbf{0 . 9 9 9}$ & 0.062 & $\mathbf{2 . 5 7 3}$ & $\mathbf{0 . 0 9 2}$ \\
\hline
\end{tabular}

Table 3. Evaluation on KITTI (Eigen split).
Zero-shot cross-dataset transfer. Table 1 shows the results of zero-shot transfer to different datasets that were not seen during training. We refer the interested reader to Ran$\mathrm{ftl}$ et al. [30] for details of the evaluation procedure and error metrics. For all metrics, lower is better. Both DPT variants significantly outperform the state of the art. The average relative improvement over the best published architecture, MiDaS, is more than 23\% for DPT-Hybrid and 28\% for DPT-Large. DPT-Hybrid achieves this with a comparable network capacity (Table 9), while DPT-Large is about 3 times larger than MiDaS. Note that both architectures have similar latency to MiDaS (Table 9).

To ensure that the observed improvements are not only due to the enlarged training set, we retrain the fullyconvolutional network used by MiDaS on our larger metadataset MIX 6. While the fully-convolutional network indeed benefits from the larger training set, we observe that both DPT variants still strongly outperform this network. This shows that DPT can better benefit from increased training set size, an observation that matches previous findings on transformer-based architectures in other fields.

The quantitative results are supported by visual comparisons in Figure 2. DPT can better reconstruct fine details while also improving global coherence in areas that are challenging for the convolutional architecture (for example, large homogeneous regions or relative depth arrangement across the image).

Fine-tuning on small datasets. We fine-tune DPT-Hybrid on the KITTI [15] and NYUv2 [35] datasets to further compare the representational power of DPT to existing work. Since the network was trained with an affine-invariant loss, its predictions are arbitrarily scaled and shifted and can have large magnitudes. Direct fine-tuning would thus be challenging, as the global mismatch in the magnitude of the predictions to the ground truth would dominate the loss. We thus first align predictions of the initial network to each training sample using the robust alignment procedure described in [30]. We then average the resulting scales and shifts across the training set and apply the average scale and 

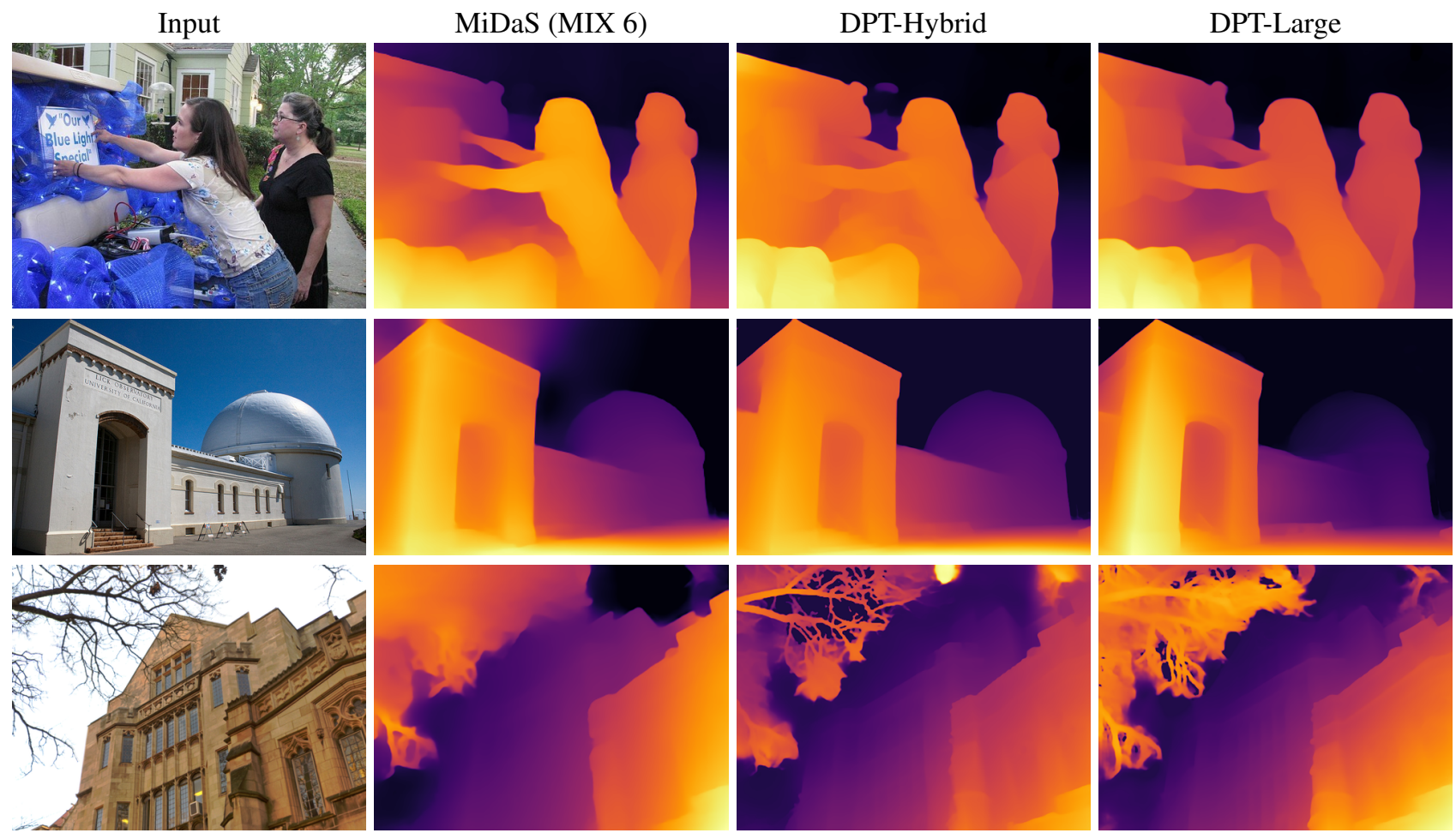

Figure 2. Sample results for monocular depth estimation. Compared to the fully-convolutional network used by MiDaS, DPT shows better global coherence (e.g., sky, second row) and finer-grained details (e.g., tree branches, last row).

shift to the predictions before passing the result to the loss. We fine-tune with the loss proposed by Eigen et al. [12]. We disable the gradient-matching loss for KITTI since this dataset only provides sparse ground truth.

Tables 2 and 3 summarize the results. Our architecture matches or improves state-of-the-art performance on both datasets in all metrics. This indicates that DPT can also be usefully applied to smaller datasets.

\subsection{Semantic Segmentation}

We choose semantic segmentation as our second task since it is representative of discrete labeling tasks and is a very competitive proving ground for dense prediction architectures. We employ the same backbone and decoder structure as in previous experiments. We use an output head that predicts at half resolution and upsamples the logits to full resolution using bilinear interpolation (details in supplementary material). The encoder is again initialized from ImageNet-pretrained weights, and the decoder is initialized randomly.

Experimental protocol. We closely follow the protocol established by Zhang et al. [51]. We employ a cross-entropy loss and add an auxiliary output head together with an auxiliary loss to the output of the penultimate fusion layer. We set the weight of the auxiliary loss to 0.2 . Dropout with a rate of 0.1 is used before the final classification layer in both heads. We use SGD with momentum 0.9 and a polynomial learning rate scheduler with decay factor 0.9 . We use batch normalization in the fusion layers and train with batch size 48 . Images are resized to 520 pixels side length. We use random horizontal flipping and random rescaling in the range $\in(0.5,2.0)$ for data augmentation. We train on square random crops of size 480 . We set the learning rate to 0.002. We use multi-scale inference at test time and report both pixel accuracy (pixAcc) as well as mean Intersectionover-Union (mIoU).

ADE20K. We train the DPT on the ADE20K semantic segmentation dataset [54] for 240 epochs. Table 4 summarizes our results on the validation set. DPT-Hybrid outperforms all existing fully-convolutional architectures. DPTLarge performs slightly worse, likely because of the significantly smaller dataset compared to our previous experiments. Figure 3 provides visual comparisons. We observe that the DPT tends to produce cleaner and finer-grained delineations of object boundaries and that the predictions are also in some cases less cluttered.

Fine-tuning on smaller datasets. We fine-tune DPTHybrid on the Pascal Context dataset [26] for 50 epochs. All other hyper-parameters remain the same. Table 5 shows results on the validation set for this experiment. We again see that DPT can provide strong performance even on smaller datasets. 

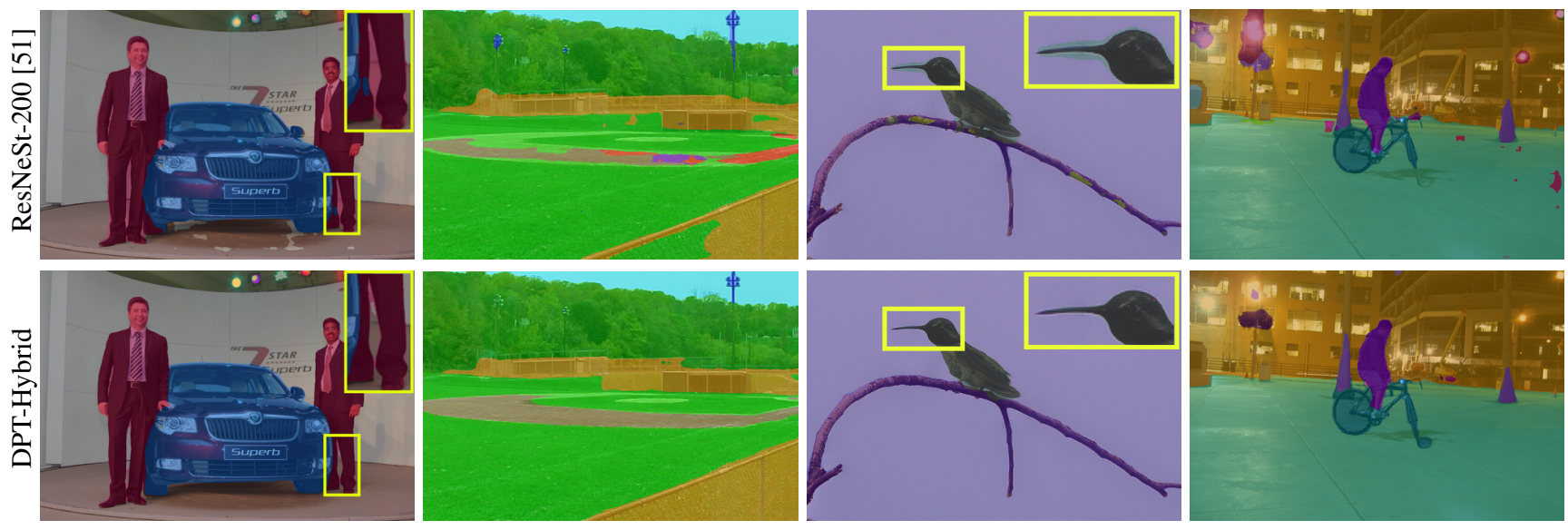

Figure 3. Sample results for semantic segmentation on ADE20K (first and second column) and Pascal Context (third and fourth column). Predictions are frequently better aligned to object edges and less cluttered.

\subsection{Ablations}

We examine a number of aspects and technical choices in DPT via ablation studies. We choose monocular depth estimation as the task for our ablations and follow the same protocol and hyper-parameter settings as previously described. We use a reduced meta-dataset that is composed of three datasets [45, 46, 47] and consists of about 41,000 images. We choose these datasets since they provide high-quality ground truth. We split each dataset into a training set and a small validation set of about 1,000 images total. We report results on the validation sets in terms of relative absolute deviation after affine alignment of the predictions to the ground truth [30]. Unless specified otherwise, we use ViT-Base as the backbone architecture.

Skip connections. Convolutional architectures offer natural points of interest for passing features from the encoder to the decoder, namely before or after downsampling of the

\begin{tabular}{lcccc}
\hline & Backbone & & pixAcc [\%] & mIoU [\%] \\
\hline OCNet & ResNet101 & {$[50]$} & - & 45.45 \\
ACNet & ResNet101 & {$[\sqrt{14}$} & 81.96 & 45.90 \\
DeeplabV3 & ResNeSt-101 & {$[7,[51$} & 82.07 & 46.91 \\
DeeplabV3 & ResNeSt-200 & {$[7,[51]$} & 82.45 & 48.36 \\
\hline DPT-Hybrid & ViT-Hybrid & & $\mathbf{8 3 . 1 1}$ & $\mathbf{4 9 . 0 2}$ \\
DPT-Large & ViT-Large & & 82.70 & 47.63 \\
\hline
\end{tabular}

Table 4. Semantic segmentation results on the ADE20K validation set.

\begin{tabular}{lcccc}
\hline & Backbone & & pixAcc [\%] & mIoU [\%] \\
\hline OCNet & HRNet-W48 & {$[42,50$} & - & 56.2 \\
DeeplabV3 & ResNeSt-200 & {$[7.51]$} & 82.50 & 58.37 \\
DeeplabV3 & ResNeSt-269 & {$[7.51$} & 83.06 & 58.92 \\
\hline DPT-Hybrid & ViT-Hybrid & & $\mathbf{8 4 . 8 3}$ & $\mathbf{6 0 . 4 6}$ \\
\hline
\end{tabular}

Table 5. Finetuning results on the Pascal Context validation set. representation. Since the transformer backbone maintains a constant feature resolution, it is not clear at which points in the backbone features should be tapped. We evaluate several possible choices in Table 6(top). We observe that it is beneficial to tap features from layers that contain low-level features as well as deeper layers that contain higher-level features. We adopt the best setting for all further experiments.

We perform a similar experiment with the hybrid architecture in Table 6(bottom), where R0 and R1 refer to using features from the first and second downsampling stages of the ResNet50 embedding network. We observe that using low-level features from the embedding network leads to better performance than using features solely from the transformer stages. We use this setting for all further experiments that involve the hybrid architecture.

Readout token. Table 7 examines various choices for implementing the first stage of the Reassemble block to handle the readout token. While ignoring the token yields good performance, projection provides slightly better performance on average. Adding the token, on the other hand, yields worse performance than simply ignoring it. We use projection for all further experiments.

Backbones. The performance of different backbones is

\begin{tabular}{|c|c|c|c|c|c|}
\hline & Layer $l$ & HRWSI & BlendedMVS & ReDWeb & Mean \\
\hline \multirow{3}{*}{$\begin{array}{l}\mathscr{D} \\
\tilde{m} \\
\mathscr{m}\end{array}$} & $\{3,6,9,12\}$ & 0.0793 & 0.0780 & 0.0892 & 0.0822 \\
\hline & $\{6,8,10,12\}$ & 0.0801 & 0.0789 & 0.0904 & 0.0831 \\
\hline & $\{9,10,11,12\}$ & 0.0805 & 0.0766 & 0.0912 & 0.0828 \\
\hline \multirow{2}{*}{$\begin{array}{l}\overline{\vec{D}} \\
\text { 永 }\end{array}$} & $\{3,6,9,12\}$ & 0.0747 & 0.0748 & 0.0865 & 0.0787 \\
\hline & $\{\mathrm{R} 0, \mathrm{R} 1,9,12\}$ & 0.0742 & 0.0751 & 0.0857 & 0.0733 \\
\hline
\end{tabular}

Table 6. Performance of attaching skip connections to different encoder layers. Best results are achieved with a combination of skip connections from shallow and deep layers. 


\begin{tabular}{l|ccc|c}
\hline & HRWSI & BlendedMVS & ReDWeb & Mean \\
\hline Ignore & $\mathbf{0 . 0 7 9 3}$ & 0.0780 & $\mathbf{0 . 0 8 9 2}$ & 0.0822 \\
Add & 0.0799 & 0.0789 & 0.0904 & 0.0831 \\
Project & 0.0797 & $\mathbf{0 . 0 7 6 4}$ & 0.0895 & $\mathbf{0 . 0 8 1 9}$ \\
\hline
\end{tabular}

Table 7. Performance of approaches to handle the readout token. Fusing the readout token to the individual input tokens using a projection layer yields the best performance.

shown in Table 8 ViT-Large outperforms all other backbones but is also almost three times larger than ViT-Base and ViT-Hybrid. ViT-Hybrid outperforms ViT-Base with a similar number of parameters and has comparable performance to the large backbone. As such it provides a good trade-off between accuracy and capacity.

ViT-Base has comparable performance to ResNext101WSL, while ViT-Hybrid and ViT-Large improve performance even though they have been pretrained on significantly less data. Note that ResNext101-WSL was pretrained on a billion-scale corpus of weakly supervised data [25] in addition to ImageNet pretraining. It has been observed that this pretraining boosts the performance of monocular depth prediction [30]. This architecture corresponds to the original MiDaS architecture.

We finally compare to a recent variant of ViT called DeIT [38]. DeIT trains the ViT architecture with a more data-efficient pretraining procedure. Note that the DeITBase architecture is identical to ViT-Base, while DeITBase-Dist introduces an additional distillation token, which we ignore in the Reassemble operation. We observe that DeIT-Base-Dist indeed improves performance when compared to ViT-Base. This indicates that similarly to convolutional architectures, improvements in pretraining procedures for image classification can benefit dense prediction tasks.

Inference resolution. While fully-convolutional architectures can have large effective receptive fields in their deepest layers, the layers close to the input are local and have small receptive fields. Performance thus suffers heavily when performing inference at an input resolution that is significantly different from the training resolution. Transformer encoders, on the other hand, have a global receptive field

\begin{tabular}{lccc|c}
\hline & HRWSI & BlendedMVS & ReDWeb & Mean \\
\hline ResNet50 & 0.0890 & 0.0887 & 0.1029 & 0.0935 \\
ResNext101-WSL & 0.0780 & 0.0751 & 0.0886 & 0.0806 \\
\hline DeIT-Base & 0.0798 & 0.0804 & 0.0925 & 0.0842 \\
DeIT-Base-Dist & 0.0758 & 0.0758 & 0.0871 & 0.0796 \\
\hline ViT-Base & 0.0797 & 0.0764 & 0.0895 & 0.0819 \\
ViT-Large & 0.0740 & 0.0747 & $\mathbf{0 . 0 8 4 6}$ & $\mathbf{0 . 0 7 7 8}$ \\
ViT-Hybrid & $\mathbf{0 . 0 7 3 8}$ & $\mathbf{0 . 0 7 4 6}$ & 0.0864 & 0.0783 \\
\hline
\end{tabular}

Table 8. Ablation of backbones. The hybrid and large backbones consistently outperform the convolutional baselines. The base architecture can outperform the convolutional baseline with better pretraining (DeIT-Base-Dist).

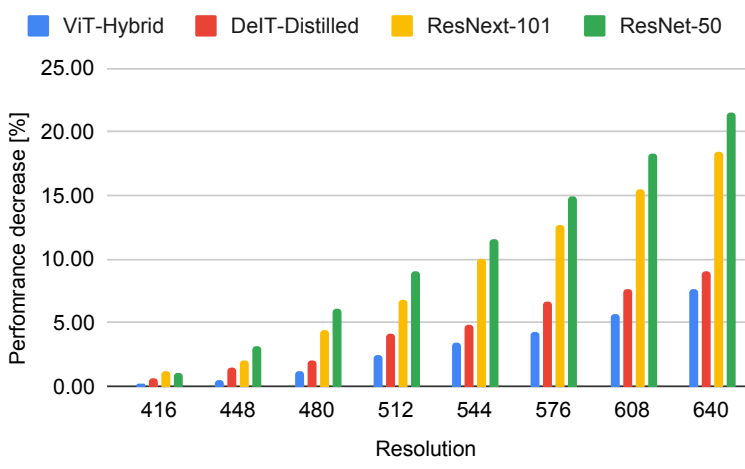

Figure 4. Relative loss in performance for different inference resolutions (lower is better).

in every layer. We conjecture that this makes DPT less dependent on inference resolution. To test this hypothesis, we plot the loss in performance of different architectures when performing inference at resolutions higher than the training resolution of $384 \times 384$ pixels. We plot the relative decrease in performance in percent with respect to the performance of performing inference at the training resolution in Figure 4 We observe that the performance of DPT variants indeed degrades more gracefully as inference resolution increases.

Inference speed. Table 9 shows inference time for different network architectures. Timings were conducted on an Intel Xeon Platinum 8280 CPU @ 2.70GHz with 8 physical cores and an Nvidia RTX 2080 GPU. We use square images with a width of 384 pixels and report the average over 400 runs. DPT-Hybrid and DPT-Large show comparable latency to the fully-convolutional architecture used by MiDaS. Interestingly, while DPT-Large is substantially larger than the other architectures in terms of parameter count, it has competitive latency since it exposes a high degree of parallelism through its wide and comparatively shallow structure.

\begin{tabular}{lcccc}
\hline & MiDaS & DPT-Base & DPT-Hybrid & DPT-Large \\
\hline Parameters [million] & 105 & 112 & 123 & 343 \\
Time [ms] & 32 & 17 & 38 & 35 \\
\hline
\end{tabular}

Table 9. Model statistics. DPT has comparable inference speed to the state of the art.

\section{Conclusion}

We have introduced the dense prediction transformer, DPT, a neural network architecture that effectively leverages vision transformers for dense prediction tasks. Our experiments on monocular depth estimation and semantic segmentation show that the presented architecture produces more fine-grained and globally coherent predictions when compared to fully-convolutional architectures. Similar to prior work on transformers, DPT unfolds its full potential when trained on large-scale datasets. 


\section{References}

[1] Vijay Badrinarayanan, Alex Kendall, and Roberto Cipolla. SegNet: A deep convolutional encoder-decoder architecture for image segmentation. IEEE TIP, 39(12):2481-2495, 2017.

[2] Dzmitry Bahdanau, Kyunghyun Cho, and Yoshua Bengio. Neural machine translation by jointly learning to align and translate. In ICLR, 2015.

[3] Irwan Bello, Barret Zoph, Ashish Vaswani, Jonathon Shlens, and Quoc V Le. Attention augmented convolutional networks. In ICCV, 2019.

[4] Tom B. Brown, Benjamin Mann, Nick Ryder, Melanie Subbiah, Jared Kaplan, Prafulla Dhariwal, Arvind Neelakantan, Pranav Shyam, Girish Sastry, Amanda Askell, et al. Language models are few-shot learners. In NeurIPS, 2020.

[5] Vincent Casser, Soeren Pirk, Reza Mahjourian, and Anelia Angelova. Unsupervised learning of depth and ego-motion: A structured approach. In $A A A I, 2019$.

[6] Liang-Chieh Chen, George Papandreou, Iasonas Kokkinos, Kevin Murphy, and Alan L. Yuille. DeepLab: Semantic image segmentation with deep convolutional nets, atrous convolution, and fully connected crfs. TPAMI, 40(4):834-848, 2018.

[7] Liang-Chieh Chen, George Papandreou, Florian Schroff, and Hartwig Adam. Rethinking atrous convolution for semantic image segmentation. arXiv preprint arXiv:1706.05587, 2017.

[8] Marius Cordts, Mohamed Omran, Sebastian Ramos, Timo Rehfeld, Markus Enzweiler, Rodrigo Benenson, Uwe Franke, Stefan Roth, and Bernt Schiele. The Cityscapes dataset for semantic urban scene understanding. In $C V P R$, 2016.

[9] Jia Deng, Wei Dong, Richard Socher, Li-Jia Li, Kai Li, and Fei-Fei Li. ImageNet: A large-scale hierarchical image database. In CVPR, 2009.

[10] Jacob Devlin, Ming-Wei Chang, Kenton Lee, and Kristina Toutanova. BERT: Pre-training of deep bidirectional transformers for language understanding. In $A C L, 2019$.

[11] Alexey Dosovitskiy, Lucas Beyer, Alexander Kolesnikov, Dirk Weissenborn, Xiaohua Zhai, Thomas Unterthiner, Mostafa Dehghani, Matthias Minderer, Georg Heigold, Sylvain Gelly, Jakob Uszkoreit, and Neil Houlsby. An image is worth 16x16 words: Transformers for image recognition at scale. arXiv preprint arXiv:2010.11929, 2020.

[12] David Eigen, Christian Puhrsch, and Rob Fergus. Depth map prediction from a single image using a multi-scale deep network. In NeurIPS, 2014.

[13] Huan Fu, Mingming Gong, Chaohui Wang, Kayhan Batmanghelich, and Dacheng Tao. Deep ordinal regression network for monocular depth estimation. In CVPR, 2018.

[14] Jun Fu, Jing Liu, Yuhang Wang, Yong Li, Yongjun Bao, Jinhui Tang, and Hanqing Lu. Adaptive context network for scene parsing. In ICCV, 2019.

[15] Andreas Geiger, Philip Lenz, and Raquel Urtasun. Are we ready for autonomous driving? The KITTI vision benchmark suite. In CVPR, 2012.

[16] Kaiming He, Xiangyu Zhang, Shaoqing Ren, and Jian Sun. Deep residual learning for image recognition. In $C V P R$, 2016.
[17] Dan Hendrycks and Kevin Gimpel. Gaussian error linear units (GELUs). arXiv preprint arXiv:1606.08415, 2016.

[18] Xinyu Huang, Peng Wang, Xinjing Cheng, Dingfu Zhou, Qichuan Geng, and Ruigang Yang. The ApolloScape open dataset for autonomous driving and its application. TPAMI, 42(10):2702-2719, 2020.

[19] Diederik P. Kingma and Jimmy Lei Ba. Adam: A method for stochastic optimization. In ICLR, 2015.

[20] Jin Han Lee, Myung-Kyu Han, Dong Wook Ko, and Il Hong Suh. From big to small: Multi-scale local planar guidance for monocular depth estimation. arXiv preprint arXiv:1907.10326, 2019.

[21] Zhengqi Li, Tali Dekel, Forrester Cole, Richard Tucker, Noah Snavely, Ce Liu, and William T. Freeman. Learning the depths of moving people by watching frozen people. In CVPR, 2019.

[22] Zhengqi Li and Noah Snavely. MegaDepth: Learning singleview depth prediction from Internet photos. In CVPR, 2018.

[23] Guosheng Lin, Anton Milan, Chunhua Shen, and Ian D. Reid. RefineNet: Multi-path refinement networks for highresolution semantic segmentation. In CVPR, 2017.

[24] Yinhan Liu, Myle Ott, Naman Goyal, Jingfei Du, Mandar Joshi, Danqi Chen, Omer Levy, Mike Lewis, Luke Zettlemoyer, and Veselin Stoyanov. RoBERTa: A robustly optimized BERT pretraining approach. arXiv preprint arXiv:1907.11692, 2019.

[25] Dhruv Mahajan, Ross Girshick, Vignesh Ramanathan, Kaiming He, Manohar Paluri, Yixuan Li, Ashwin Bharambe, and Laurens van der Maaten. Exploring the limits of weakly supervised pretraining. In ECCV, 2018.

[26] Roozbeh Mottaghi, Xianjie Chen, Xiaobai Liu, Nam-Gyu Cho, Seong-Whan Lee, Sanja Fidler, Raquel Urtasun, and Alan L. Yuille. The role of context for object detection and semantic segmentation in the wild. In $C V P R, 2014$.

[27] Hyeonwoo Noh, Seunghoon Hong, and Bohyung Han. Learning deconvolution network for semantic segmentation. In ICCV, 2015.

[28] Niki Parmar, Ashish Vaswani, Jakob Uszkoreit, Lukasz Kaiser, Noam Shazeer, Alexander Ku, and Dustin Tran. Image transformer. In ICML, 2018.

[29] Prajit Ramachandran, Niki Parmar, Ashish Vaswani, Irwan Bello, Anselm Levskaya, and Jonathon Shlens. Stand-alone self-attention in vision models. In NeurIPS, 2019.

[30] René Ranftl, Katrin Lasinger, David Hafner, Konrad Schindler, and Vladlen Koltun. Towards robust monocular depth estimation: Mixing datasets for zero-shot cross-dataset transfer. TPAMI, 2020.

[31] Olaf Ronneberger, Philipp Fischer, and Thomas Brox. UNet: Convolutional networks for biomedical image segmentation. In MICCAI, 2015.

[32] Ozan Sener and Vladlen Koltun. Multi-task learning as multi-objective optimization. In NeurIPS, 2018.

[33] Pierre Sermanet, David Eigen, Xiang Zhang, Michaël Mathieu, Rob Fergus, and Yann LeCun. OverFeat: Integrated recognition, localization and detection using convolutional networks. In ICLR, 2014.

[34] Evan Shelhamer, Jonathan Long, and Trevor Darrell. Fully convolutional networks for semantic segmentation. CVPR, 2015.

[35] Nathan Silberman, Derek Hoiem, Pushmeet Kohli, and Rob 
Fergus. Indoor segmentation and support inference from RGBD images. In $E C C V, 2012$.

[36] Josef Sivic and Andrew Zisserman. Efficient visual search of videos cast as text retrieval. TPAMI, 31(4):591-606, 2009.

[37] Ke Sun, Bin Xiao, Dong Liu, and Jingdong Wang. Deep high-resolution representation learning for human pose estimation. In CVPR, 2019.

[38] Hugo Touvron, Matthieu Cord, Matthijs Douze, Francisco Massa, Alexandre Sablayrolles, and Hervé Jégou. Training data-efficient image transformers \& distillation through attention. arXiv preprint arXiv:2012.12877, 2020.

[39] Ashish Vaswani, Noam Shazeer, Niki Parmar, Jakob Uszkoreit, Llion Jones, Aidan N. Gomez, Lukasz Kaiser, and Illia Polosukhin. Attention is all you need. In NeurIPS, 2017.

[40] Chaoyang Wang, Oliver Wang, Federico Perazzi, and Simon Lucey. Web stereo video supervision for depth prediction from dynamic scenes. In 3DV, 2019.

[41] Huiyu Wang, Yukun Zhu, Bradley Green, Hartwig Adam, Alan L. Yuille, and Liang-Chieh Chen. Axial-DeepLab: Stand-alone axial-attention for panoptic segmentation. In ECCV, 2020.

[42] Jingdong Wang, Ke Sun, Tianheng Cheng, Borui Jiang, Chaorui Deng, Yang Zhao, Dong Liu, Yadong Mu, Mingkui Tan, Xinggang Wang, Wenyu Liu, and Bin Xiao. Deep high-resolution representation learning for visual recognition. TPAMI, 2020.

[43] Qiang Wang, Shizhen Zheng, Qingsong Yan, Fei Deng, Kaiyong Zhao, and Xiaowen Chu. IRS: A large synthetic indoor robotics stereo dataset for disparity and surface normal estimation. arXiv preprint arXiv:1912.09678, 2019.

[44] Wenshan Wang, Delong Zhu, Xiangwei Wang, Yaoyu Hu, Yuheng Qiu, Chen Wang, Yafei Hu, Ashish Kapoor, and Sebastian Scherer. TartanAir: A dataset to push the limits of visual slam. In IROS, 2020.

[45] Ke Xian, Chunhua Shen, Zhiguo Cao, Hao Lu, Yang Xiao, Ruibo Li, and Zhenbo Luo. Monocular relative depth perception with web stereo data supervision. In $C V P R, 2018$.

[46] Ke Xian, Jianming Zhang, Oliver Wang, Long Mai, Zhe Lin, and Zhiguo Cao. Structure-guided ranking loss for single image depth prediction. In $C V P R, 2020$.

[47] Yao Yao, Zixin Luo, Shiwei Li, Jingyang Zhang, Yufan Ren, Lei Zhou, Tian Fang, and Long Quan. BlendedMVS: A large-scale dataset for generalized multi-view stereo networks. CVPR, 2020.

[48] Wei Yin, Yifan Liu, Chunhua Shen, and Youliang Yan. Enforcing geometric constraints of virtual normal for depth prediction. In $I C C V, 2019$.

[49] Fisher Yu and Vladlen Koltun. Multi-scale context aggregation by dilated convolutions. In ICLR, 2016.

[50] Yuhui Yuan, Xilin Chen, and Jingdong Wang. Objectcontextual representations for semantic segmentation. In ECCV, 2020.

[51] Hang Zhang, Chongruo Wu, Zhongyue Zhang, Yi Zhu, Zhi Zhang, Haibin Lin, Yue Sun, Tong He, Jonas Muller, R. Manmatha, Mu Li, and Alexander Smola. ResNeSt: Splitattention networks. arXiv preprint arXiv:2004.08955, 2020.

[52] Hengshuang Zhao, Jiaya Jia, and Vladlen Koltun. Exploring self-attention for image recognition. In CVPR, 2020.

[53] Hengshuang Zhao, Jianping Shi, Xiaojuan Qi, Xiaogang Wang, and Jiaya Jia. Pyramid scene parsing network. In
CVPR, 2017.

[54] Bolei Zhou, Hang Zhao, Xavier Puig, Sanja Fidler, Adela Barriuso, and Antonio Torralba. Scene parsing through ADE20K dataset. In $C V P R, 2017$. 


\section{Supplementary Material}

\section{A. Architecture details}

We provide additional technical details in this section.

Hybrid encoder. The hybrid encoder is based on a preactivation ResNet50 with group norm and weight standardization [57]. It defines four stages after the initial stem, each of which downsamples the representation before applying multiple ResNet blocks. We refer by $R N$ to the output of the $N$-th stage. DPT-Hybrid thus taps skip connections after the first $(R 0)$ and second stage $(R 1)$.

Residual convolutional units. Figure A1 (a) shows a schematic overview of the residual convolutional units [23] that are used in the decoder. Batch normalization is used for semantic segmentation but is disabled for monocular depth estimation. When using batch normalization, we disable biases in the preceding convolutional layer.

Monocular depth estimation head. The output head for monocular depth estimation is shown in Figure A1 (b). The initial convolution halves the feature dimensions, while the second convolution has an output dimension of 32 . The final linear layer projects this representation to a non-negative scalar that represent the inverse depth prediction for every pixel. Bilinear interpolation is used to upsample the representation.

Semantic segmentation head. The output head for semantic segmentation is shown in Figure A1 (c). the first convolutional block preserves the feature dimension, while the final linear layer projects the representation to the number of output classes. Dropout is used with a rate of 0.1 . We use bilinear interpolation for the final upsampling operation. The prediction thus represents the per-pixel logits of the classes.

\section{B. Additional results}

We provide additional qualitative and quantitative results in this section.

Monocular depth estimation. We notice that the biggest gains in performance for zero-shot transfer were achieved for datasets that feature dense, high-resolution evaluations [15, 55, 59]. This could be explained by more finegrained predictions. Visual inspection of sample results (c.f. Figure A3) from these datasets confirms this intuition.
We observe more details and also better global depth arrangement in DPT predictions when compared to the fullyconvolutional baseline. Note that results for DPT and MiDaS are computed at the same input resolution (384 pixels).

Semantic segmentation. We show per-class IoU scores for the ADE20K validation set in Figure A2. While we observe a general trend of an improvement in per-class IoU in comparison to the baseline [51], we do not observe a strong pattern across classes.

Attention maps. We show attention maps from different encoder layers in Figures A4 and A5. In both cases, we show results from the monocular depth estimation models. We visualize the attention of two reference tokens (upper left corner and lower right corner, respectively) to all other tokens in the image across various layers in the encoder. We show the average attention over all 12 attention heads.

We observe the tendency that attention is spatially more localized close to the reference token in shallow layers (leftmost columns), whereas deeper layers (rightmost columns) frequently attend across the whole image.

\section{References}

[55] D. J. Butler, J. Wulff, G. B. Stanley, and M. J. Black. A naturalistic open source movie for optical flow evaluation. In ECCV, 2012.

[56] Andreas Geiger, Philip Lenz, and Raquel Urtasun. Are we ready for autonomous driving? The KITTI vision benchmark suite. In CVPR, 2012.

[57] Alexander Kolesnikov, Lucas Beyer, Xiaohua Zhai, Joan Puigcerver, Jessica Yung, Sylvain Gelly, and Neil Houlsby. Big transfer (bit): General visual representation learning. In ECCV, 2020.

[58] Guosheng Lin, Anton Milan, Chunhua Shen, and Ian D. Reid. RefineNet: Multi-path refinement networks for highresolution semantic segmentation. In CVPR, 2017.

[59] Thomas Schöps, Johannes L. Schönberger, Silvano Galliani, Torsten Sattler, Konrad Schindler, Marc Pollefeys, and Andreas Geiger. A multi-view stereo benchmark with highresolution images and multi-camera videos. In CVPR, 2017. 


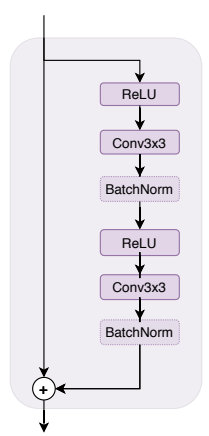

(a) Residual Convolutional Unit [23]

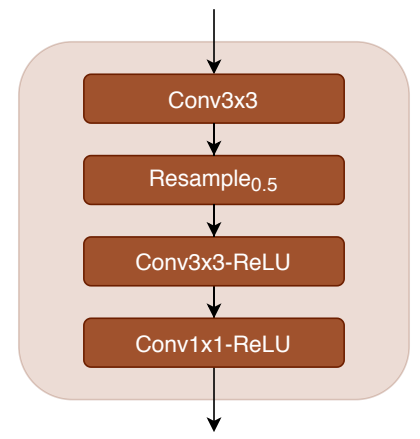

(b) Monocular depth estimation head

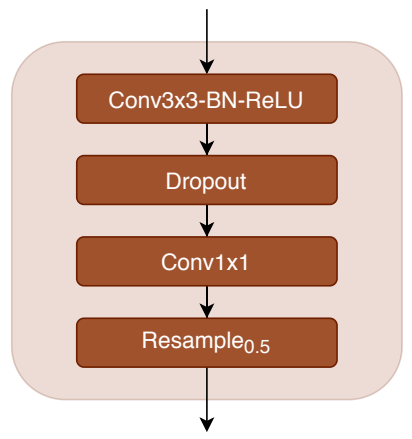

(c) Semantic segmentation head

Figure A1. Schematics of different architecture blocks.

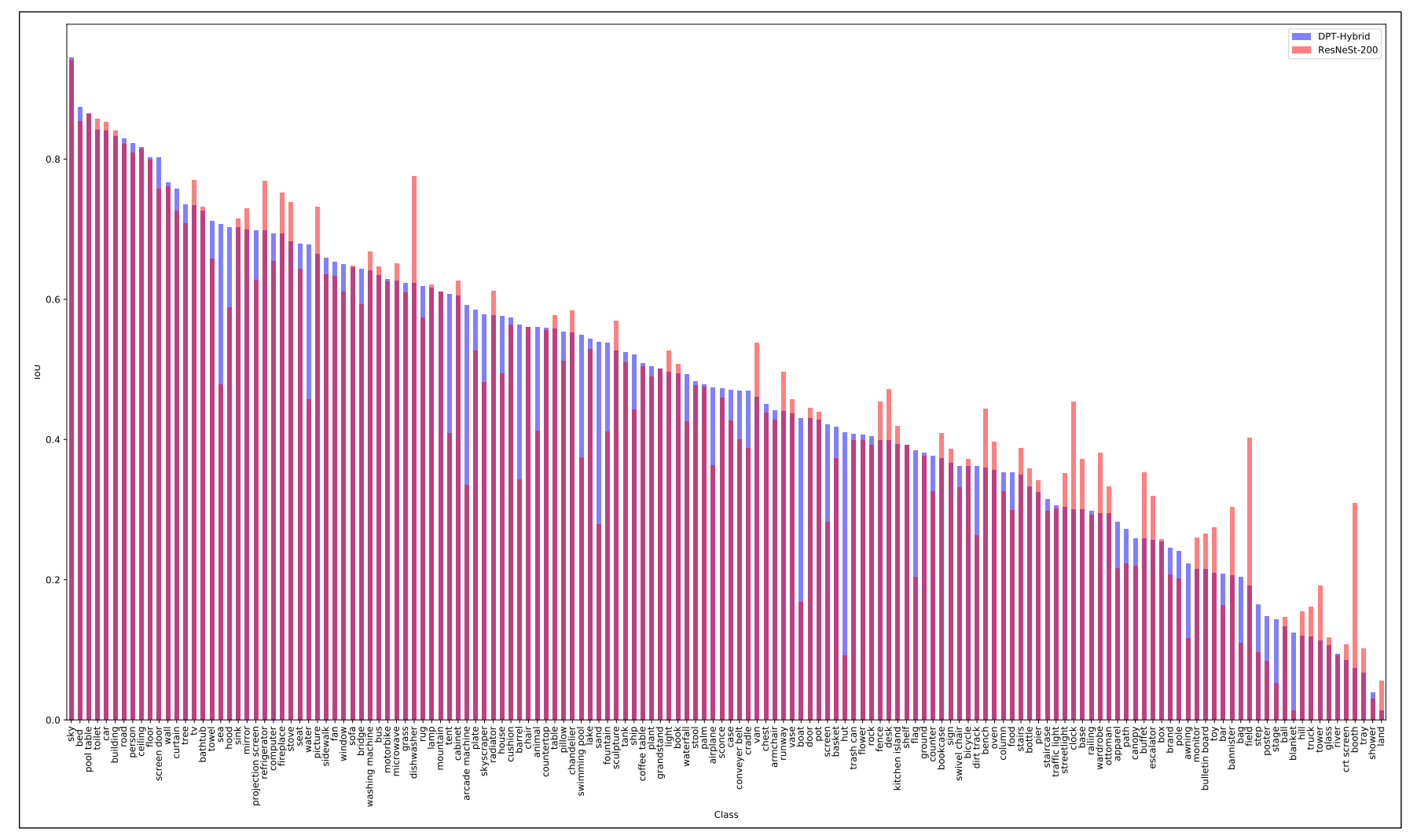

Figure A2. Per class IoU on ADE20K. 


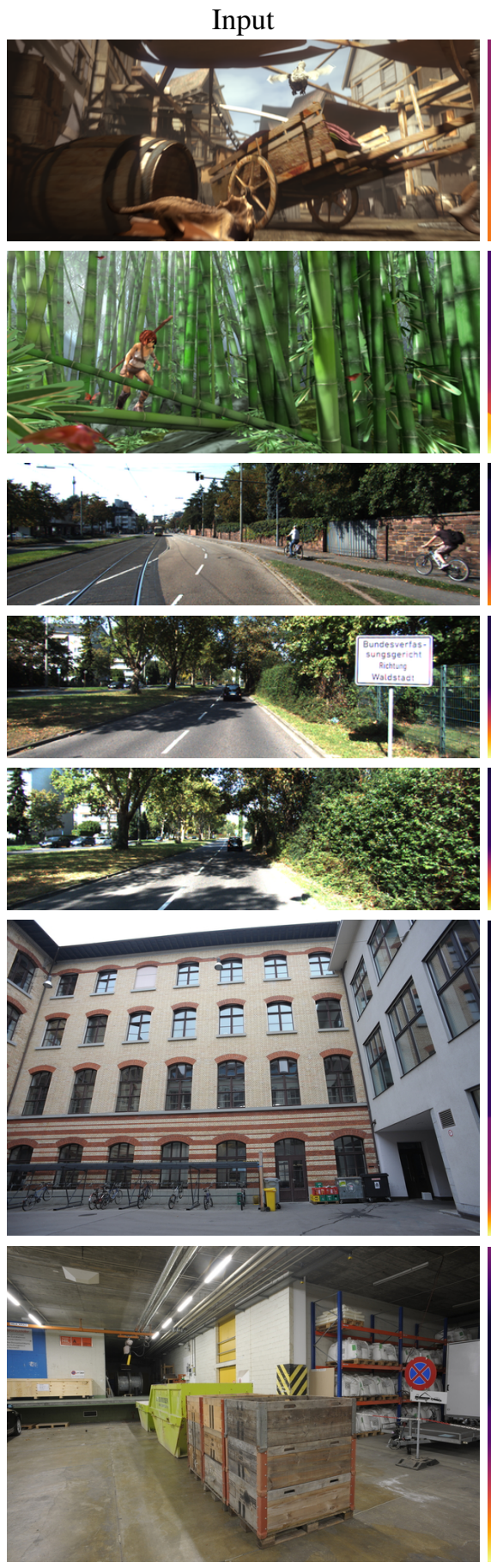

MiDaS (MIX 5)
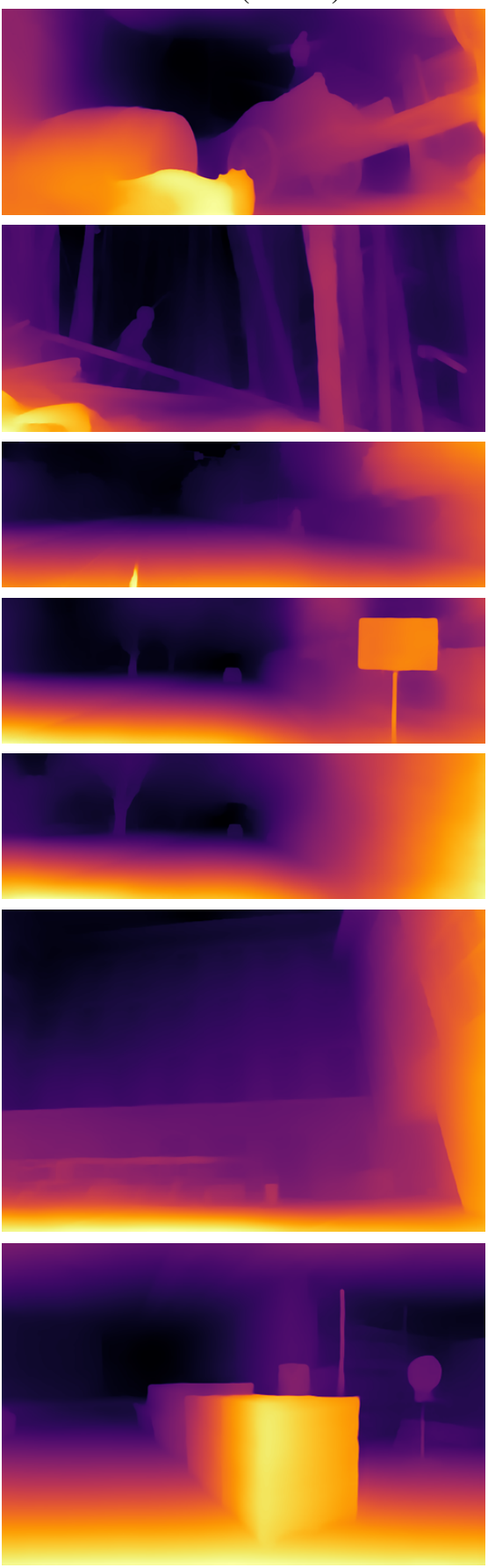

DPT-Large
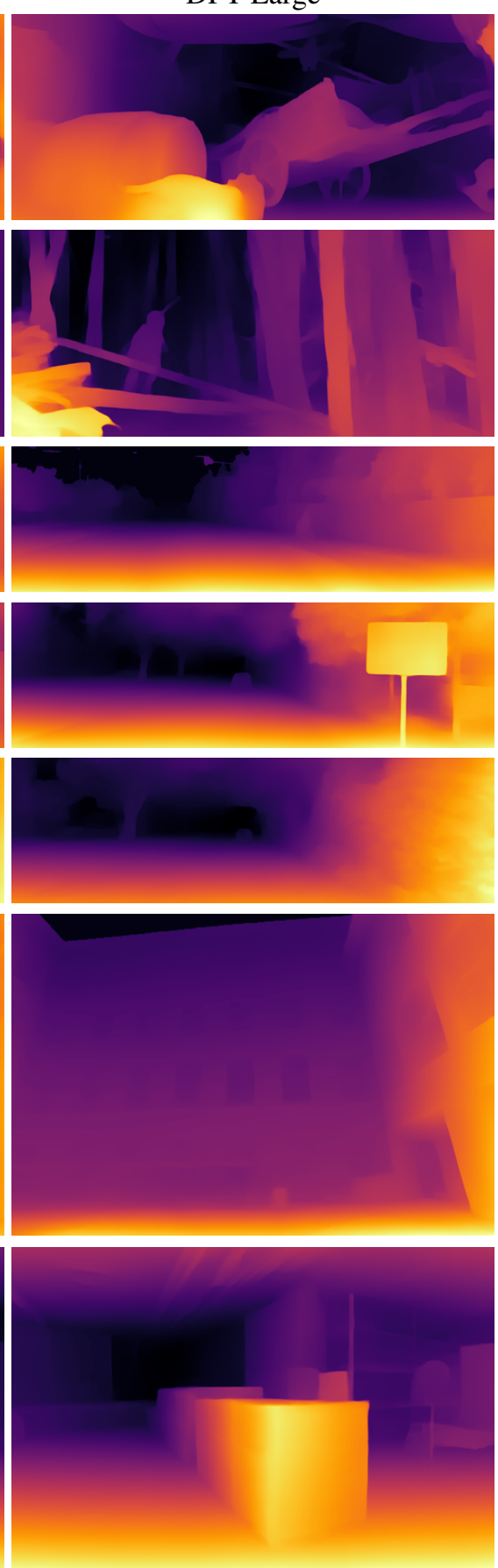

Figure A3. Additional comparisons for monocular depth estimation. 
Input

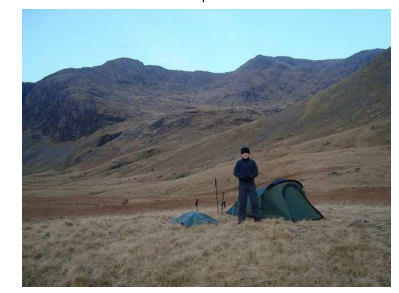

Layer 6
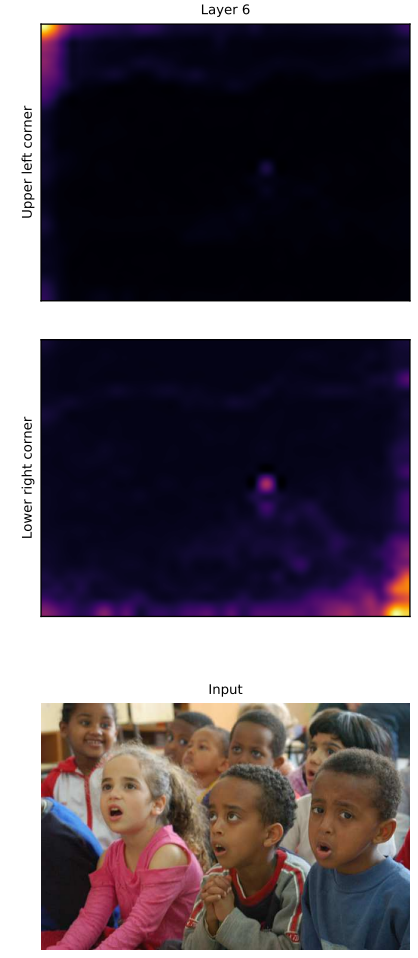

Layer 6
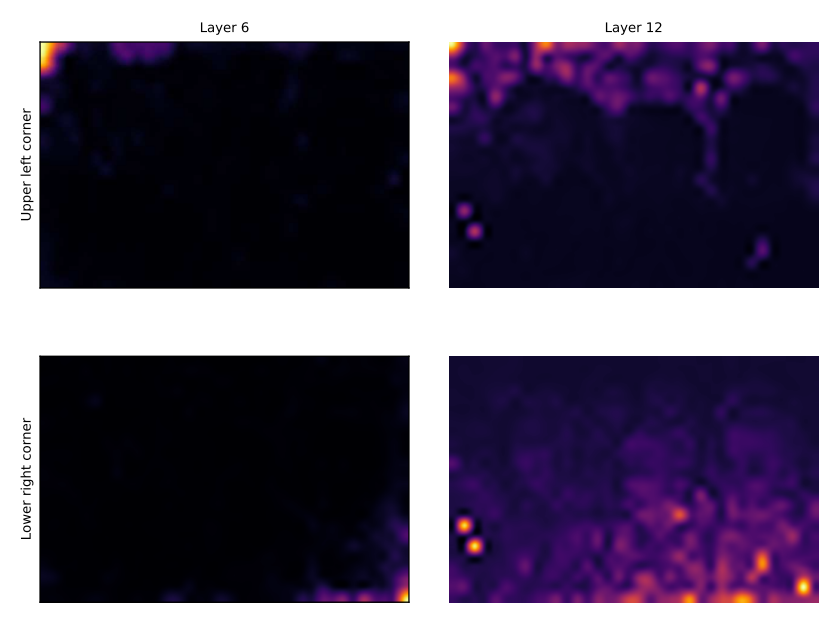

Prediction

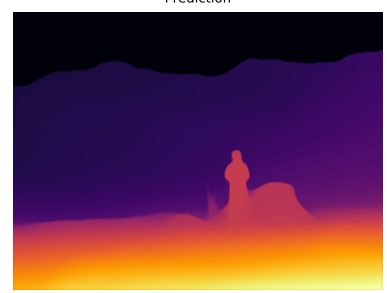

Layer 12
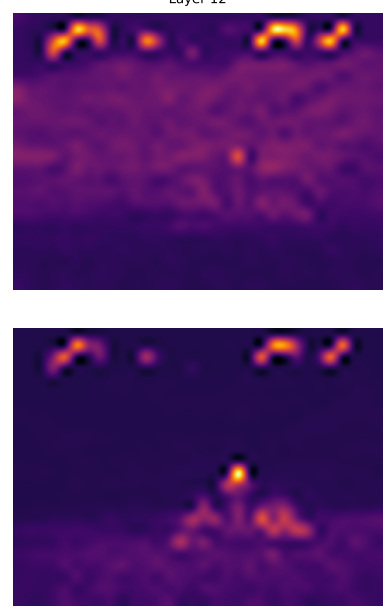

Prediction
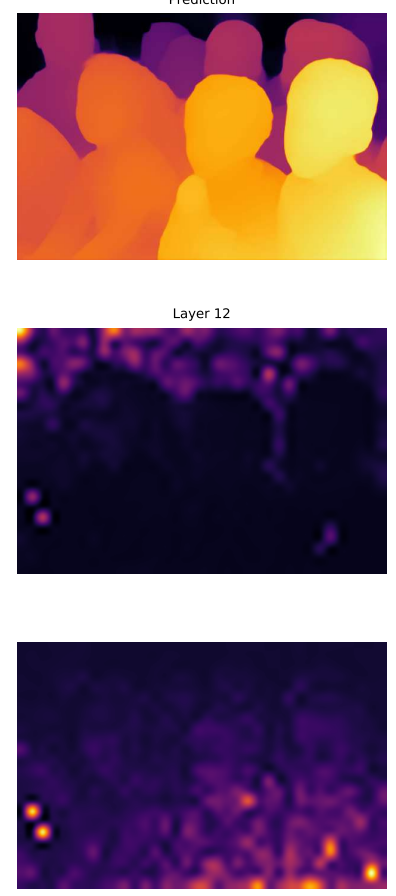
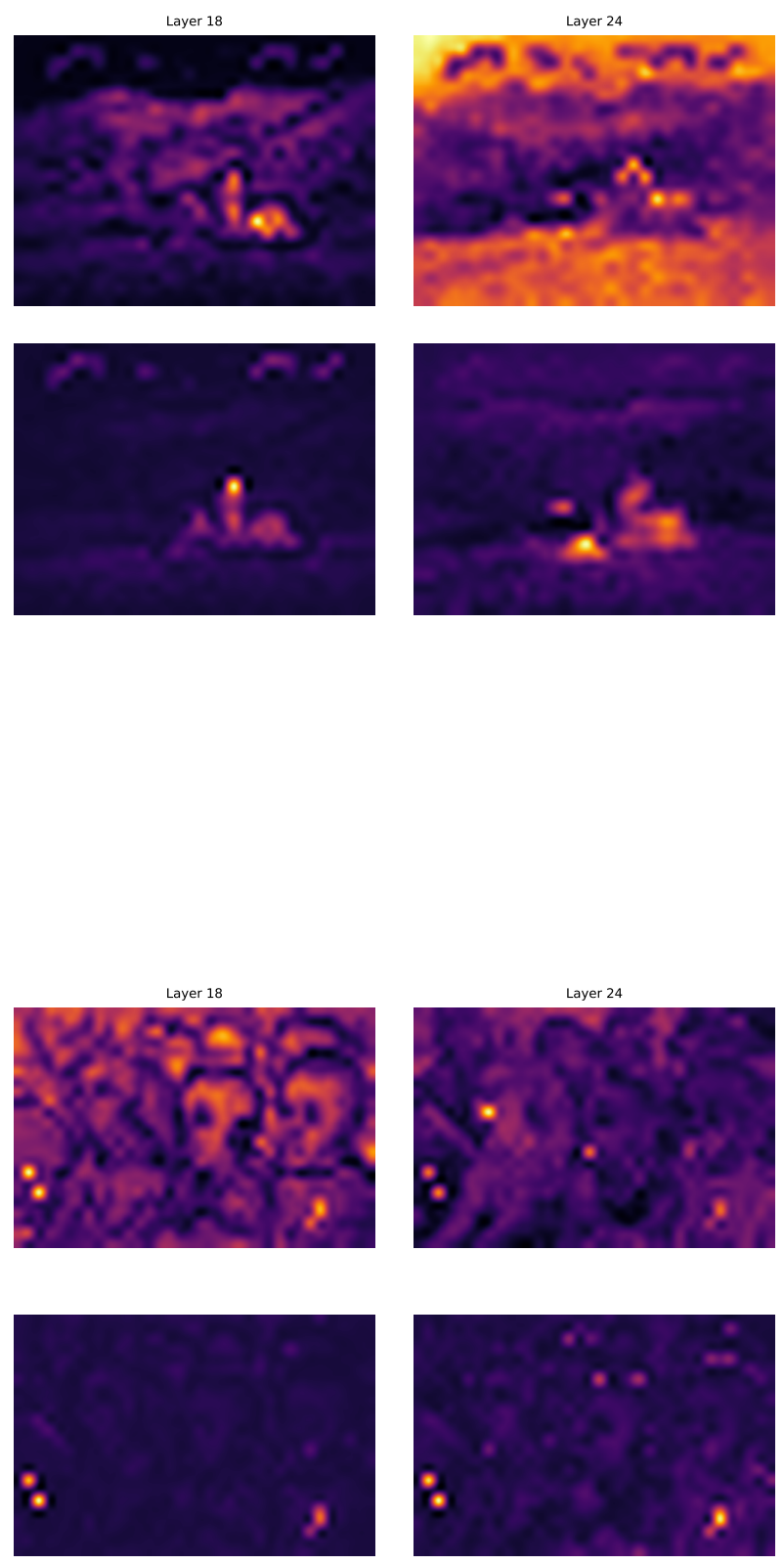

Figure A4. Sample attention maps of the DPT-Large monocular depth prediction network. 
Input

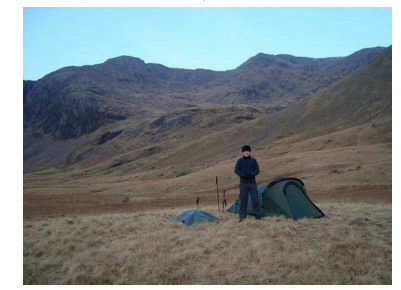

Layer 3
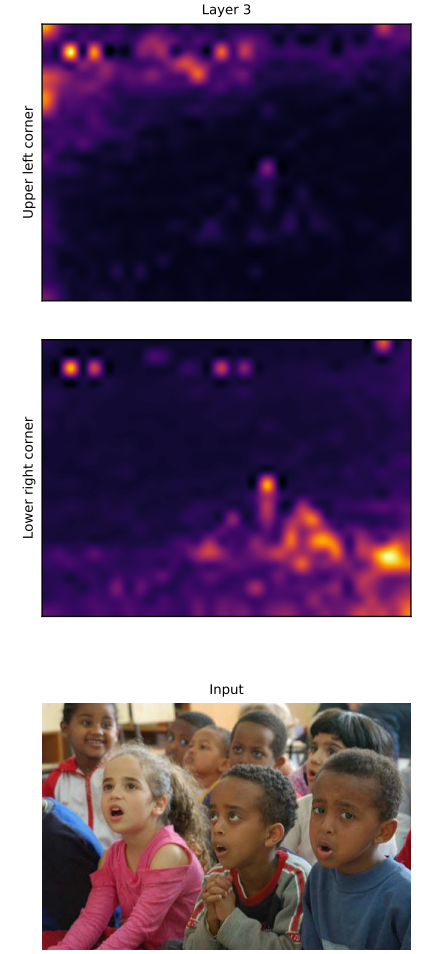

Layer 3
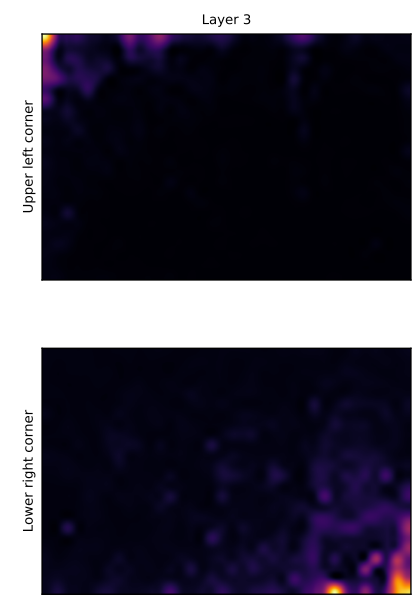

Prediction

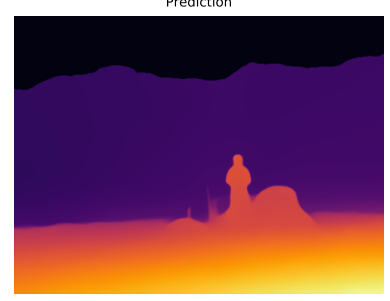

Layer 6
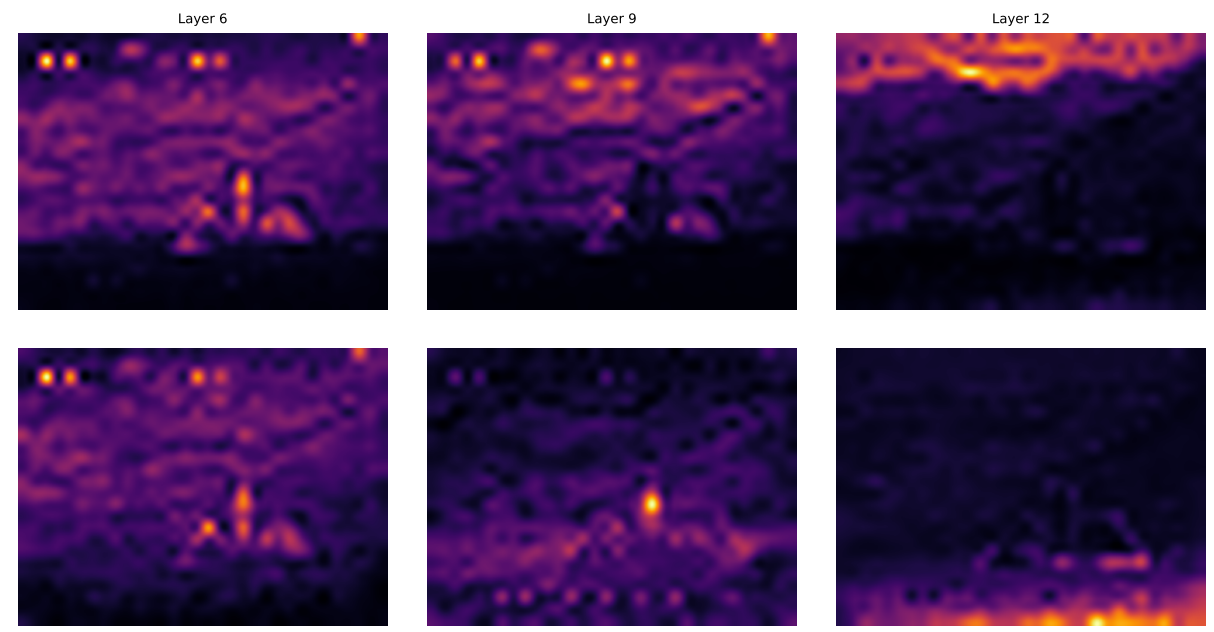

Prediction

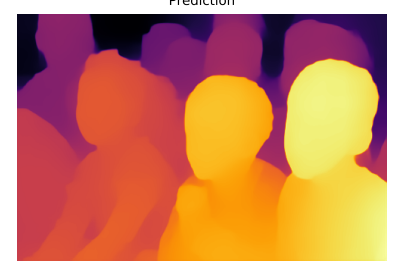

Layer 6
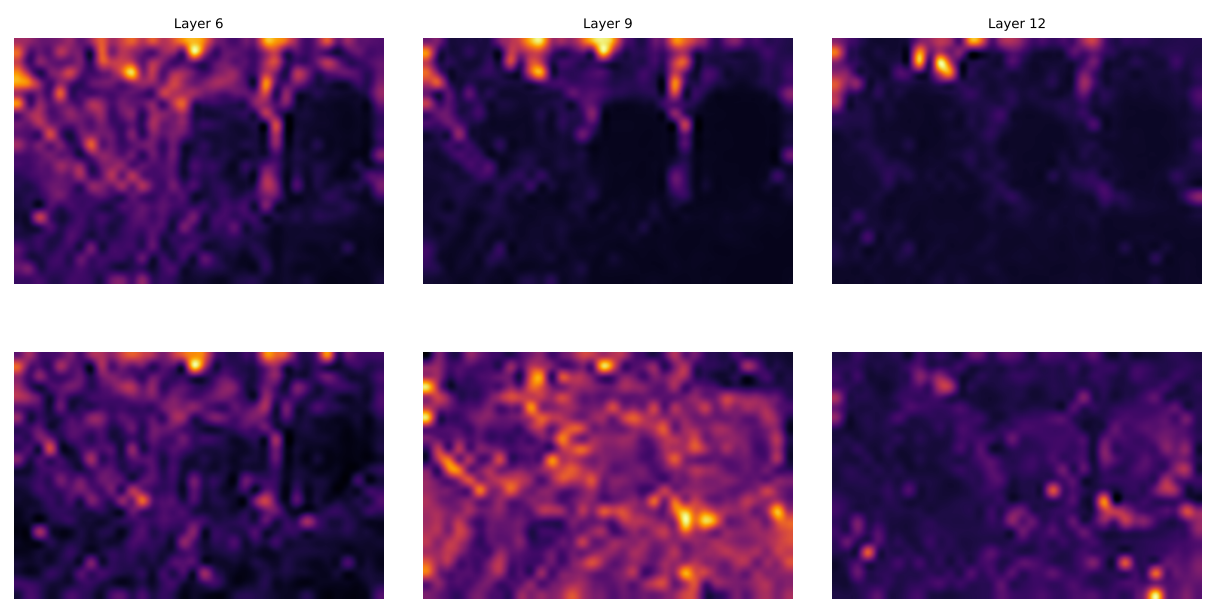

Figure A5. Sample attention maps of the DPT-Hybrid monocular depth prediction network. 